\title{
Эллипсометрическая in situ диагностика температуры в технологии выращивания слоёв кадмий-ртуть-теллур методом молекулярно-лучевой эпитаксии
}

\author{
В.А. Швец ${ }^{1,2}$, Д.В. Марин ${ }^{1}$, И.А. Азаров ${ }^{1}$, М.В. Якушев ${ }^{1}$, С.В. Рыхлицкий ${ }^{1}$ \\ ${ }^{1}$ Институт физики полупроводников им. А.В. Ржанова СО РАН, 630090, г. Новосибирск, Россия. \\ ${ }^{2}$ Новосибирский государственный университет, 630090, г. Новосибирск, Россия.
}

эл.nочта: basi5353@mail.ru

DOI 10.34077/RCSP2021-90

Температура подложки при выращивании слоёв кадмий-ртуть-теллур (КРТ) в технологии молекулярно-лучевой эпитаксии является одним из ключевых параметров, который обеспечивает стабильный рост бездефектных эпитаксиальных плёнок с заданными характеристиками. Используемые в мировой практике методы контроля температуры, такие как пирометрия, спектроскопия края поглощения, термопарный либо малоэффективны, либо требуют дополнительной технической адаптации вакуумного оборудования.

В ИФП СО РАН применяются эллипсометрические методики диагностики процессов эпитаксии КРТ. В данном сообщении представлены последние достижения в области разработки спектральных эллипсометрических методов контроля температуры при выращивании структур МЛЭ КРТ. С методической точки зрения можно выделить три стадии процесса: 1) подготовка к росту подложки CdTe и выход на заданный температурный режим; 2) начальный этап роста, слой КРТ остаётся полупрозрачным; 3) стационарный этап роста, зондирующее излучение отражается только от поверхности КРТ. Для каждого из этих этапов реализуются индивидуальные методы решения поставленной задачи.

Для стадии подготовки к росту разработаны две методики, использующие температурную зависимость края поглощения СdTe, определяемого по началу интерференционных осцилляций, и температурную зависимость спектров оптических постоянных CdTe в области его поглощения. Тестовые измерения и последующие эксперименты показали, что первая из этих методик имеет чувствительность, составляющую несколько градусов Цельсия и высокую устойчивость к систематическим погрешностям измерений и углу падения света. В противоположность этому вторая методика имеет высокую обнаружительную способность, но чувствительна к углу падения. Сочетание этих двух методик обеспечивает возможность отслеживать динамику слабых температурных изменений с амплитудой, не превышающей $5^{\circ} \mathrm{C}$.

На второй стадии роста оптическая модель оказывается наиболее сложной, так как содержит большое число переменных. Это оптические постоянные и толщина $\mathrm{CdTe}$, оптические постоянные и изменяющаяся толщина КРТ, а также его состав. Между тем, именно на этой стадии происходят наиболее сильные изменения условий, которые могут повлиять на температуру: открытие молекулярных источников, создающих дополнительный поток теплового излучения, изменение излучательной способности образца. На сегодняшний день есть только предварительные пути решения поставленной задачи на этом этапе.

На стадии стационарного роста эллипсометрические спектры зависят только от температуры и состава КРТ, и задача заключается в независимом определении обоих параметров. Для этого была разработана разностная методика сравнения спектров, измеренных в разные моменты роста. Изменение состава $\delta$ и и температуры $\delta \mathrm{T}$ на выбранном временном интервале находится путём минимизации функционала:

$$
S_{\Psi}(\delta x, \delta T)=\sum_{\lambda}\left[\Psi(\lambda)-\Psi_{0}(\lambda)-\frac{\partial \Psi}{\partial T} \delta T+\frac{\partial \Psi}{\partial x} \delta x\right]^{2},
$$

где $\Psi(\lambda)$ и $\Psi_{0}(\lambda)$ - спектры параметра $\Psi$, измеренные в начале и в конце интервала. Спектральные зависимости фигурирующих здесь частных производных были измерены экспериментально и представлены в параметрическом виде.

Проверка предложенной методики проводилась для двух условий роста: с изменением в процессе роста температурного режима нагревателя и при постоянном напряжении на нагревателе. В первом случае оказалось, что вместе с падением температуры уменьшается также и состав растущего слоя. Во втором случае выяснилось, что, несмотря на постоянство режима нагревателя, происходит слабое возрастание температуры роста на 2 - $4^{\circ}$. Возрастание температуры наблюдается также и по показаниям термопары, расположенной в зоне нагревателя, что подтверждает корректность эллипсометрического метода. Наблюдаемое изменение температуры связано скорее всего с тепловым потоком от молекулярных источников. 